\title{
Guidelines for Management of Neurosurgical Cases during COVID-19 Pandemic
}

\author{
Raj Kumar $^{1}$ Ashok Kumar Mahapatra ${ }^{2}$ Radhe Shyam Mittal ${ }^{3}$ Hanuman Prasad Prajapati ${ }^{4}$
}

\author{
${ }^{1}$ Uttar Pradesh University of Medical Sciences (UPUMS), Etawah, \\ Uttar Pradesh, India \\ ${ }^{2}$ SOA deemed to be University, Bhubaneswar, Odisha, India \\ ${ }^{3}$ Department of Neurosurgery, All India Institute of Medical \\ Sciences, Rishikesh, Uttarakhand, India \\ ${ }^{4}$ Department of Neurosurgery, Uttar Pradesh University of Medical \\ Sciences (UPUMS), Etawah, Uttar Pradesh, India
}

\author{
Address for correspondence Prof. Raj Kumar, MCh (Neurosurgery), \\ Vice Chancellor, Uttar Pradesh University of Medical Sciences \\ (UPUMS), Saifai, Etawah, Uttar Pradesh 206130, India \\ (e-mail: rajkumar1959@gmail.com).
}

Indian J Neurosurg 2019;8:3-7

\begin{abstract}
Transmission of Coronavirus Disease 2019 (COVID-19) infection can occur to health care workers while providing treatment. It is vital to ensure the safety and to avoid any undue exposure of health care workers, leading to collapse of the entire health care system. As the world is facing this grave threat due to COVID-19, a proper pro-

Keywords

- COVID 19

- neurosurgical procedures

- guidelines tocol should be there. Daily updates and modifications are being made available by the Ministry of Health \& Family Welfare and the ministry advises that protocols be tailored as per prevailing local conditions. We are trying to lay down guidelines based on available literature, data, and guidelines to take the procedures of neurosurgery and neurosciences.
\end{abstract}

\section{Introduction}

In December 2019, Coronavirus Disease 2019 (COVID-19) was discovered in Wuhan, Hubei province, and has spread rapidly to various countries. This has caused serious harm to people's health and a huge economic burden. ${ }^{1}$ Therefore, it is pertinent to curtail the infection, rationalize the use of resources, and manage the patient rush in the given scenario.The typical clinical manifestation of COVID-19 positive patients are fever, cough, and dyspnea. However, several patients present with neurological symptoms, such as vomiting, headache, dizziness, and delirium. Anosmia and ageusia can also be early symptoms., ${ }^{2,3}$

\section{Protocol for Patients with Central Nervous System Disease}

Patients with diseases of the central nervous system could be approached according to the timing needed for surgery.

(A) Patients requiring immediate intervention: These patients require immediate intervention within 24 hours. Examples: all trauma cases requiring immediate intervention, intracranial tumors with sudden deterioration, ruptured aneurysm, pituitary adenoma with visual deterioration, prolapsed intervertebral disc with cauda equina syndrome, and hydrocephalus requiring immediate intervention.

(B) Patients requiring early intervention: These patients require early intervention within 1 week. Example: large tumors like meningiomas, gliomas with decompensating symptoms, arteriovenous malformations, and spinal tumors with progressive deficits.

(C) Patients requiring late intervention: These patients require intervention within a month. Example: schwannoma,pituitary adenoma (without visual deterioration), craniopharyngioma, lumbar canal stenosis (-Fig. 1).

\section{Protocol for Neurosurgical Patients Requiring Early/ Late Intervention}

Avoid use of drills/craniotomies (it is associated with significant aerosol formation).

(1) Use Hudson's brace and Gigli saw.

(2) Copious saline irrigations during craniotomies. 


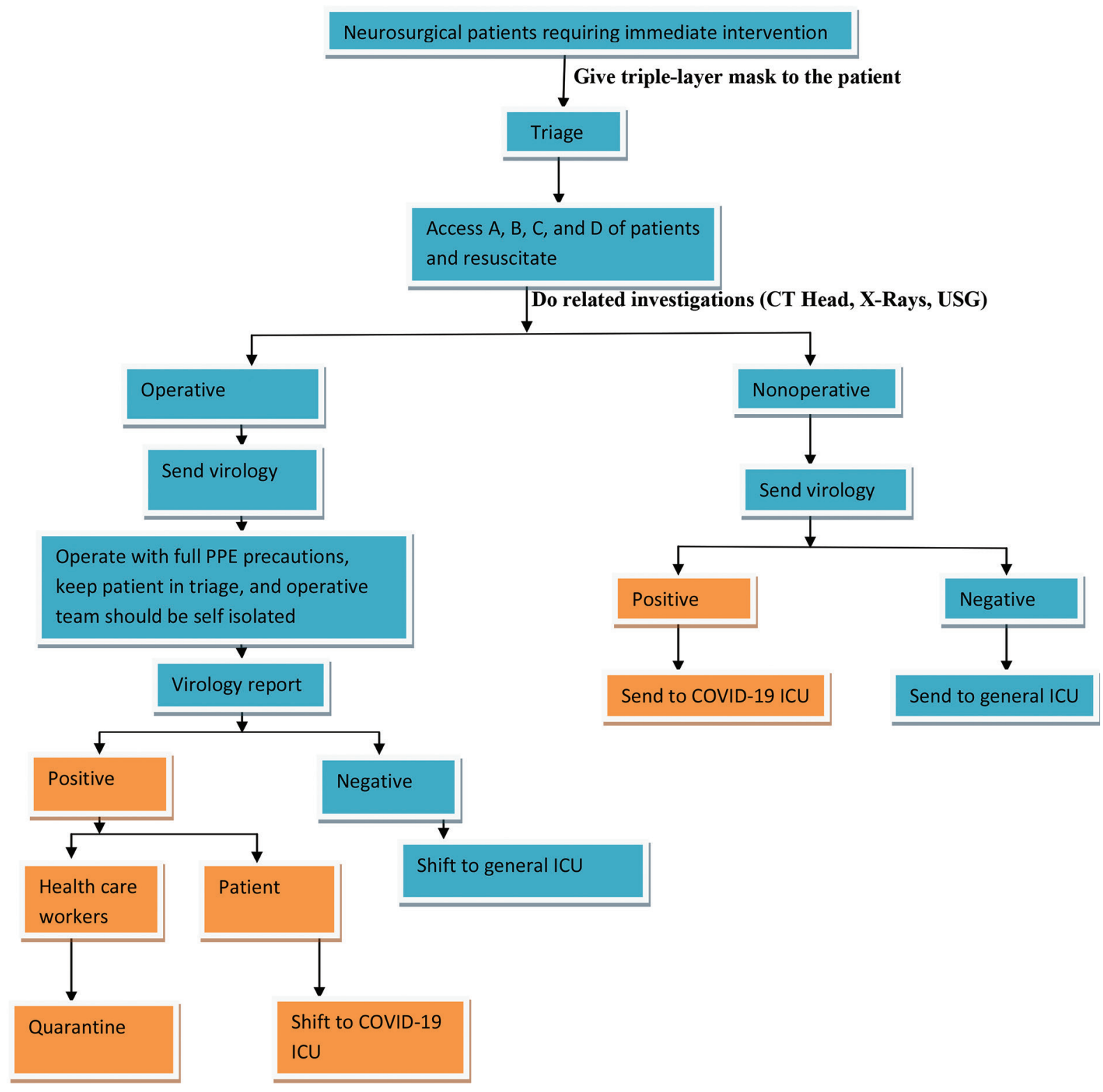

Protocol for neurosurgical patients requiring early/late intervention:

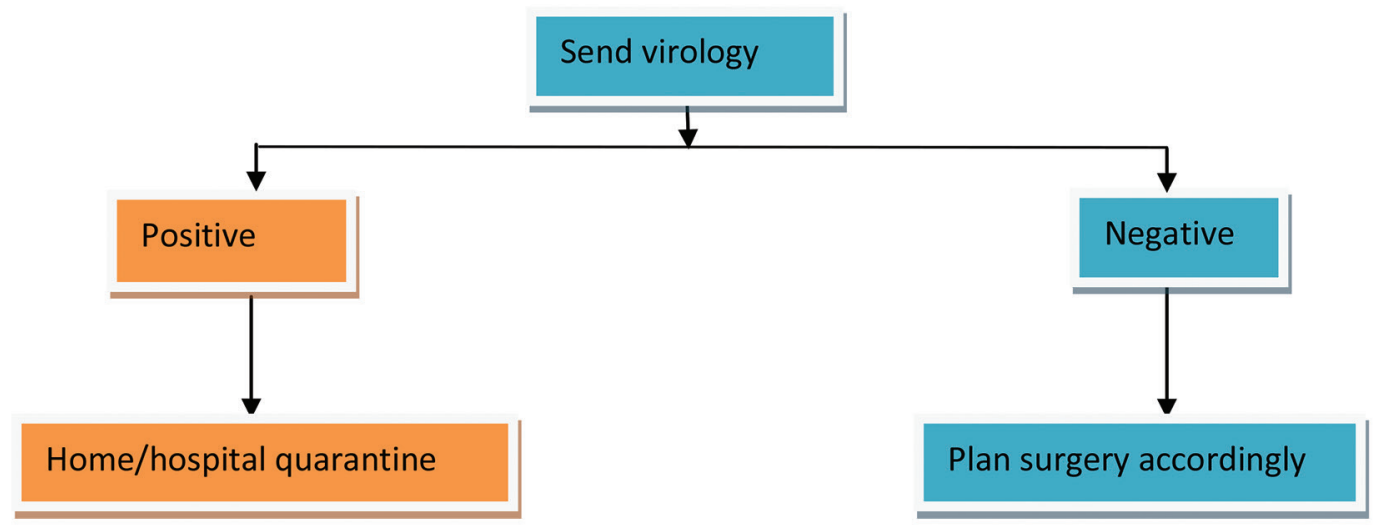

Fig. 1 Protocol for neurosurgery patients suspected with COVID-19 needing surgery. ${ }^{4-6}$ ICU, intensive care unit; PPE, personal protection equipment; USG, ultrasonography. 
(3) If drills are used during spinal/cranial procedures, they must be used at lower speeds and with liberal irrigation to reduce the risk of aerosol contamination of the theater milieu.

\section{Protocols for Endonasal Procedures}

Endonasal procedures are at high risk of transmission. The use of debriders and drills within the nasal cavity will produce a highly dangerous droplet aerosol. It is recommended to avoid sinus breach whenever possible and avoid the use of any instrument which causes aerosol droplets to form.

\section{Protocol for Postoperative Management}

(1) Postoperative RT-PCR of throat swab are recommended in all cases.

(2) All postoperative patients should be assumed to be suspected cases and quarantined for at least 2 weeks.

(3) RT-PCR of throat swab should be repeated three times in 2 weeks. If the tests are negative after 2 weeks, the quarantine may be terminated and patients may be shifted to the ward or an individual room.

\section{Protocols for Neurosurgery Operation Theater (OT)}

(1) There should be a separate OT for COVID-19 positive and negative patients.

(2) Follow Donning and Doffing protocol step wise.

(3) There should be separate area for Donning and Doffing.

(4) The neurosurgeons, anesthetists, nurses, and paramedical staff must take strict protection before entering the OT.

(5) OT fumigation should be done after each case.

\section{Protocol for Treatment of Spinal Disease during COVID-19 Pandemic}

\section{(A) Nonoperative treatment:}

Conservative treatment (including prolonged bed rest) increases the risk of respiratory and urinary tract infections. Thus, surgeons should weigh the advantages and disadvantages of conservative treatment and adopt the most appropriate treatment methods. Moreover, patients can use online follow-up to minimize unnecessary hospital visits. Patients whose symptoms have not improved significantly after a period of standardized conservative treatment can be admitted to the hospital for surgery after the epidemic is under control. ${ }^{7}$

\section{(B) Operative treatment:}

Emergency spinal operation requires for patients with prolapsed intervertebral disc with cauda equina syndrome, spinal cord injury with quadri- or paraparesis, spinal fracture with obvious displacement or compression, and spinal cord tumors with deterioration. ${ }^{7}$
(1) Proper protective precautions should be used by operative team.

(2) Try to use minimally invasive surgery and shorten the operation time.

(3) Prone position is preferred to avoid viral transmission by respiratory droplets.

(4) Reduce the use of electrotomes under the principle of ensuring complete hemostasis.

(5) Suction devices should be used with caution to reduce aerosol diffusion.

(6) The operation should be gentle and careful to avoid body fluid spatter and sharp instrument injury.

(7) Operation personnel should be screened for the virus before and after an operation, and anyone with suspicious symptoms should be isolated for medical treatment in time.

\section{Protocols for Neurosurgery Outpatient Department (OPD)}

(1) Ensure thermal screening and isolation of suspected patient.

(2) There should be separate entry for doctors and patients.

(3) There should be a glass barrier between patient and doctor.

(4) Surgeons should wear hair covers, gloves, face-masks (Respirator N95 or equivalent), and eye protection (goggles or face shield, better if disposable).

(5) Alcohol-based hand sanitizer with 60 to $95 \%$ alcohol should be used after attending each patient.

(6) Avoiding any paper reports or MRI films. It is important to switch over to electronic medical records and visualizing MRI and other imaging using a CD or through a hospital intranet server facility.

(7) Avoid overcrowding in OPD.

\section{Protocol for Neurosurgery Ward Rounds}

(1) Surgeons should wear head cover, shoe covers, facemask (Respirator N95 or equivalent), gown, and eye protection goggles or face shield.

(2) Hand hygiene should be maintained after attending to each patient.

(3) The distance between each bed should be around 6 feet for the general ward or the patients should be lodged in separate rooms to prevent cross-infection.

(4) Triple layers mask should be given to every patients.

\section{Sample Collection}

(1) Preferred sample: Throat and nasal swab in viral transport media (VTM) and transported on ice.

(2) Alternate: Nasopharyngeal swab, bronchoalveolar lavage, or endotracheal aspirate which has to be mixed with the viral transport medium and transported on ice. 8 


\section{General Guidelines}

- Trained health care professionals to wear appropriate PPE with latex-free purple nitrile gloves while collecting the sample from the patient.

- Restricted entry to visitors or attendants during sample collection.

- Complete the requisition form for each specimen submitted.

- Proper disposal of all waste generated.

\section{Specific Therapy}

No specific antivirals have been proven to be effective as per currently available data.

However, based on the available information (uncontrolled clinical trials), the following drugs maybe considered as an off-label indication in patients with severe disease and requiring ICU management.

- Hydroxychloroquine (Dose 400mg BD-for 1 day followed by $200 \mathrm{mg}$ BD for 4 days)

In combination with

- Azithromycin (500 mg OD for 5 days)

Hydroxychloroquine can be used as prophylaxis for symptomatic health care worker. ${ }^{9}$ These drugs should be administered under close medical supervision, with monitoring for side effects including QTc interval.

The above medication is presently not recommended for children less than 12 years, pregnant and lactating women.These guidelines are based on currently available information and would be reviewed from time to time as new evidence emerges.

\section{Protocol for Discharge}

(1) Patient should remain in quarantine for next 14 days at home.

(2) Patient should report to COVID-19 screening center, if there is a recurrence of symptoms.

(3) If any relative at home becomes symptomatic (fever, cough or breathlessness), he/she should report to COVID-19 screening center.

(4) Medications for seizures and comorbidities should be continued.

(5) Medicines may be prescribed for symptomatic relief.

\section{Protocols for Health Care Workers in Hospital}

(1) Take minimum accessories in hospital duty (like purse, pen, bag, belt, keys, mobile, charger, laptop, etc.).

(2) Use face mask in proper way and change it timely.

(3) Cover head with surgeon's cap.

(4) Cover eye with goggles.

(5) Use double gloves with proper protocol.

(6) Avoid to take tea and breakfast in ward.

\section{Protocols for Health Care Workers at Home}

On reaching home, perform the following advice:

(1) Remove clothes in outer room of your home and soak it in hot water with detergent.

(2) Drink warm water 2 to3 times a day.

(3) Sanitize mobile charger, switches, and remotes routinely.

(4) Maintain social distancing in-house also.

(5) Follow standard operating procedures (SOP) strictly.

(6) Use turmeric, ginger, garlic, cinnamon, basil as spices.

(7) Do household exercises.

\section{Bio-medical Waste Management}

(1) All PPEs, face masks, and gloves worn during sampling to be incinerated (yellow bin). Rest of all plastic should be autoclaved (red bin).

(2) Separate laundry facility should be available.Wherever possible, disposable sheet should be used.

(3) Laundry of active quarantined to be soaked in $0.5 \%$ hypochlorite for 30 minutes followed by washing with hot water $\left(70^{\circ} \mathrm{C}\right)$.

(4) Standard precautions must also include prevention of injury, safe waste management, cleaning and disinfection of equipment.

(5) Environmental cleaning is a part of precaution and ensures disinfection procedure consistently and correctly. ${ }^{10}$

\section{Conclusion}

Transmission of COVID-19 can occur to health care workers while providing emergency treatments. There should be proper guidelines to manage the neurosurgical cases and to save the whole team. It helps to prevent the intrahospital transmission of COVID-19 infections.

\section{Conflict of interest}

None declared.

\section{References}

1 World Health Organization. US\$675 million needed for new coronavirus preparedness and response global plan [Internet]. Geneva: World Health Organization; 2020. [cited 2020 Feb 5]. Available from: https://www.who.int/ news-room/detail/05-02-2020-us-675-million-needed-fornew-coronavirus-preparedness-and-response-global-plan. Accessed May 5, 2020

2 Gaunt ER, Hardie A, Claas EC, Simmonds P, Templeton KE. Epidemiology and clinical presentations of the four human coronaviruses 229E, HKU1, NL63, and OC43 detected over 3 years using a novel multiplex real-time PCR method. J Clin Microbiol 2010;48(8):2940-2947 
3 Huang C, Wang Y, Li X, et al. Clinical features of patients infected with 2019 novel coronavirus in Wuhan, China. Lancet 2020;395(10223) :497-506

4 Ministry of Health \& Family Welfare. Revised Guidelines on Clinical Management of COVID-19. Available at: https://www.mohfw. gov.in/pdf/RevisedNationalClinicalManagementGuidelinefor COVID1931032020.pdf. Accessed March 31, 2020

5 American College of Surgeons. COVID-19: Elective Case Triage Guidelines for Surgical Care. Published March 24, 2020. Available at: https://www.facs.org/covid-19/clinical-guidance/elective-case. Accessed March 27, 2020

6 Ministry of Health \& Family Welfare. Guidance document on appropriate management of suspectirmed cases of COVID 19. Available at: https://www. mohfw.gov.in/pdf/FinalGuidanceonMangaementof Covidcasesversion2.pdf. Accessed on March 31, 2020
7 Li Y, Li ZF, Mao QX, et al. Consensus on emergency surgery and infection prevention and control for severe trauma patients with 2019 novel coronavirus pneumonia. J Chin J Trauma 2020;36:97-103

8 Ministry of Health \& Family Welfare. Guidance on specimen collection, processing, transportation, including related biosafety procedures. Available at: https://mohfw.gov.in/media/ disease-alerts. Accessed March 31, 2020

9 P, LagierJC, ParolaP, et al. Hydroxychloroquine and azithromycin as a treatment of COVID-19: Results of an open-label non-randomized clinical trial. Int J Antimicrob Agents 2020:105949. [epub ahead of print] doi: 10.1016/j. ijantimicag.2020.105949

10 World Health Organization. Hand Hygiene for Health Workers Caring for Ebola Patients. Available at: https://www.who.int/csr/ disease/ebola/hand-hygiene/en/. Accessed February 7, 2020 